\title{
Comparison of glucose yield from rubberwood sawdust (RSD), growth medium (GM), and mushroom spent medium (MSM) under different sodium hydroxide pretreatment techniques
}

\author{
Siti Aminah Mohd Hassan 1,*, Muhammad Syarhabil Ahmad ${ }^{2}$, Amira Farzana Samat ${ }^{1}$, Nur Zatul 'Iffah \\ Zakaria $^{1}$, Khairunissa Syairah Ahmad Sohaimi ${ }^{1}$, Nurfatirah Nordin ${ }^{1}$ \\ ${ }^{1}$ Department of Chemical Engineering Technology, Universiti of Malaysia Perlis, 02100 Padang Besar, Perlis, Malaysia. \\ ${ }^{2}$ School of Bioprocess Engineering, Universiti of Malaysia Perlis, 02600 Arau, Perlis, Malaysia
}

\begin{abstract}
Large quantities of oyster mushroom (Pleurotus sajor caju. )spent medium (MSM) were disposed of as agricultural waste to the environment and thereby constituting environmental pollution. Therefore the study was conducted to examine the feasibility of using MSM as glucose source in comparison to RSD and GM under different $\mathrm{NaOH}$ pretreatment techniques and at the same enzymatic saccharification condition. The composition analysis proved that decreases in lignocellulosic contents occurred after cultivation of The amount of lignin, hemicelluloses, and cellulose in MSM showed lower values than those in RSD and GM which were 22.40, 27.93, and $27.97 \%$ respectively. The surface morphology of MSM appeared to be rough and broken and traces of hyphen on the fibre surface were observed as a result from the mushroom cultivation process. RSD and FM had an even and smooth flat surface, indicating a rigid and highly ordered surface structure. The highest glucose yield were obtained from MSM under all three $\mathrm{NaOH}$ pretreatment techniques (autoclaving at $121^{\circ} \mathrm{C}$, heating in water bath, soaking at room temperature), that were $31.07,30.13$, and $21.97, \mathrm{~g} /$ dry substrate respectively. The amount of glucose obtained from RSD and GM under the same treatments were comparable.
\end{abstract}

\section{Introduction}

Glucose from sugar crops (sugarcane, sugar beets) and starchy food (potato, cassava, corn) can be converted into ethanol via fermentation process with the assist of microbes or enzymes. However, concerns about its production and use related to the increased food prices due to the large amount of arable land required for crops, as well as the energy and pollution balance of the whole cycle of ethanol production has caused a new source of glucose being introduced such as lignocellulosic biomass that may allay these concerns. Concomitantly, over the last decades, research efforts have been devoted to converting the promising feedstock of lignocellulosic biomass into biofuel, especially residues from agricultural and forestry operations due to its great availability, sustainability, and low cost compared to other energy feedstock [1].

The rubber tree or scientific name "Hevea brasiliensis" is one of the major agricultural crops grown in Malaysia besides oil palm, cocoa, rice, and coconut. The trees are logged off after 25 to 30 years and utilized mainly for making furnitures. As a result of logging and lumber processes, large amount of residual biomass were generated, which have no significant value except its usage in the making of products like briquetted fuel and compressed powder boards [2]. However, due to its high cellulose content, rubber wood waste represents a potential raw material for bioethanol production [3]. Apart from its potential as bioethanol feedstock, rubberwood sawdust maybe used to cultivate oyster mushroom and then, further used the remaining medium wastes to produce glucose for bioethanol production. Extra income could be generated and necessary pretreatment steps could be reduced prior to the enzymatic hydrolysis process with high reducing sugars yield via the aforementioned processes. According to previous study, lignocellulosic biomass, which has been biologically treated by fungi have been proven to increase the sugar produced during enzymatic saccharafication and lower lignin contents was detected as a result from the fully/partially digested lignocellulosic materials. Wan and Li has reported that, with the result of selective delignification, enzymatic digestibility as high as $60-80 \%$ has been obtained from fungal-pretreated corn stover, which was comparable to that obtained from chemical pretreatment [4]. Unlike biologically pretreated biomass, the raw lignocellulosic biomass could not be saccharified by enzymes for high glucose yield without first undergoing several pretreatment including, physical, mechanical, and chemical pretreatment. The main cause for the recalcitrance of lignocellulosic biomass is the presence of lignin and hemicelluloses on the surface of cellulose. They formed a barrier and prevented cellulase from accessing the cellulose in the substrate [5]. The

* Corresponding author: aminahhassanr@unimap.edu.my 
pretreatment of lignocellulosics was primarily employed to increase the accessible surface area of cellulose to enhance the conversion of cellulose to glucose in enzymatic saccharification.

Pretreatment of lignocellulosic materials was considered as the rate-limiting step in an economically feasible process for enzymatic hydrolysis of cellulose. Combination of biological and chemical pretreatment was expected to increase the cellulose amount, which could be feasibly accessed by cellulase in subsequent enzymatic saccharification, after removing the lignin content. Apparently, to produce ethanol from cellulosic feedstock was more costly than to use other starchy source such as corn due to the present of lignin structure in lignocellulosic biomass that form barrier from enzymatic susceptibility. Punsuvan mentioned that, main contributors to the overall costs of bioethanol refinery were the raw materials, pretreatment, and enzyme production [6]. Therefore, it would be worthwhile to explore the potential of mushroom spent medium with partially/ fully delignified structure, which might acquired less intermediate pretreatment steps and low amount of enzyme loading for high glucose yield, as a new second generation energy feedstock. Therefore this study is conducted to explore the potential of MSM in producing high glucose yoeld compared to RSD and GM under different sodium hydroxide pretreatment techniques.

\section{Materials and Methods}

\subsection{Preparation of biomass samples}

$\mathrm{RSD}, \mathrm{GM}$, and MSM were oven-dried at $100^{\circ} \mathrm{C}$ for 48 hours and then stored in an air tight container at room temperature until further use. The substrates with size of 254-510 $\mu \mathrm{m}$ were used in this study.

\subsection{Analysis of lignocellulosic contents}

The lignocellulosic contents analysis of the RSD, GM, and MSM were carried out in five stages. First of all, the ash content was analyzed according to American Standard Test Method (ASTM). Then, the extractives were removed from biomass samples followed by removal of hemicelluloses via dissolution using dilute $\mathrm{NaOH}$ solution [7]. The next stage involved the dissolution of lignin using $\mathrm{H}_{2} \mathrm{SO}_{4}$ dilution method. Finally, the celluloses were calculated as the difference of ash, extractives, hemicelluloses and lignin from 100\% of total lignocellulosic content in biomass samples. Extractives, hemicelluloses, lignin, and cellulose content were determined by aforementioned methods as proposed by $\mathrm{Li}$ et al. [8]. All analytical experiments were performed in triplicates and the average values were presented.

\subsection{Analysis of morphology surface}

Examinations of the microstructure surface morphologies of RSD, GM, and MSM were carried out at School of Material Engineering, UniMAP. A thin layer of gold was coated on the samples prior to measurement to prevent charging on the surface. SEM micrographs with 1000x magnifications were employed to observe the morphologies of RSD, GM and MSM samples.

\section{3 $\mathrm{NaOH}$ pretreatment techniques}

\subsubsection{Autoclaving at $121^{\circ} \mathrm{C}$ for $20 \mathrm{~min}$}

Sample concentrations of $5 \%(\mathrm{w} / \mathrm{v})$ with particle size in the range of 254-500 $\mu \mathrm{m}$ were treated in an autoclave at $121^{\circ} \mathrm{C}$ for 20 minutes. As a control, extra samples with the same concentration were immersed in distilled water. After autoclaving process completed, the treated samples were washed extensively over running water using $45 \mu \mathrm{m}$ sieve tray until the $\mathrm{pH}$ reached 7 followed by final rinse with distilled water to remove excess $\mathrm{NaOH}$ and by-products that might inhibited the cellulase activity in subsequent enzymatic saccharification. The hydrolysates were, then, dried in an oven at $60^{\circ} \mathrm{C}$ for 24 hours before further use.

\subsection{Heating in water bath $\left(30,60,90^{\circ} \mathrm{C}\right)$ for $2 h$}

In the second pretreatment method, the same sample concentrations and particle size as Section 3.5.1.1 were heated in different concentration of $\mathrm{NaOH}(0.5,1.0,1.5$, and $2.0 \mathrm{M}$ ) at different temperature of $30,60,90^{\circ} \mathrm{C}$ for 2 hours with sample immersed in distilled water as control. The samples recovery and drying process were similar to the previous autoclaving pretreatment method.

\subsubsection{Soaking at room temperature for $2 h$}

As for soaking method, the same sample concentrations and particle size as Section 3.5.1.1 were immersed in $0.5,1.0,1.5$, and $2.0 \mathrm{M}$ of $\mathrm{NaOH}$ at room temperature for 2 hours with sample in distilled water as control. The samples recovery and drying process were performed as previous autoclaving pretreatment method.

\subsection{Enzymatic saccharafication}

The efficiency and feasibility of the aforementioned pretreatment methods were determined by total glucose yield obtained from the pretreated samples in enzymatic saccharification. One gram of hydrolysates from different pretreated RSD, GM, and MSM was immersed in $100 \mathrm{ml}$ of $50 \mathrm{mM}$ sodium citrate buffer ( $\mathrm{pH} 4.8$ ) (Leustean et al., 2010) in a $250 \mathrm{ml}$ Erlenmeyer flask, to give a final sample concentration of $1 \%(\mathrm{w} / \mathrm{v})$. One 
mililiter of cellulase from Trichoderma reesei, which its activity (67 FPU/ml) has been previously determined, was added and the samples mixtures were incubated at $50^{\circ} \mathrm{C}$ with rate of agitation at $150 \mathrm{rpm}$ for $48 \mathrm{~h}$. After the saccharification time had elapsed, the flasks were heated at $90^{\circ} \mathrm{C}$ for 10 minutes to deactivate the enzyme [9]. Then, a total volume of $2.0 \mathrm{ml}$ sample mixture was taken out for centrifugation at $8000 \mathrm{rpm}$ for $10 \mathrm{~min}$. The supernatant was filtered through $0.45 \mu \mathrm{m}$ nylon membrane filter before analyzing the glucose contents using Biochemistry Analyzer, YSI 2900. The glucose analyzed was reported in gram per 100 grams dry substrate and was calculated according to the Equation 3.1 as proposed by [10]. It should be noted that dry substrate referred to the state of the hydrolysates sample after $\mathrm{NaOH}$ pretreatment prior to enzymatic saccharification.

Glucose yield ( $\mathrm{g} / 100 \mathrm{~g}$ dry substrate)

$$
=\left(\mathrm{C}^{*} \mathrm{~V}\right) /(\mathrm{W}) \times 100 \mathrm{~g}
$$

where, $\mathrm{C}$ was the concentration of glucose $(\mathrm{g} / \mathrm{l})$, V was the total volume of the liquid phase (1), and $\mathrm{W}$ was the dry weight of the corresponding lignocellulosic waste (g).

\section{Results and Discussion}

\subsection{Lignocellulosic contents}

Table 3.1 shows the chemical components; in particular of ash, extractives, hemicelluloses, lignin, and cellulose contents in rubberwood sawdust (RSD), growth medium (GM), and mushroom spent medium (MSM). Among these three sample types, RSD contained the highest amount of lignin $(29.30 \% \pm 1.31)$, which was slightly higher than in GM $(29.07 \% \pm 0.72)$, regardless the previous performed steaming pretreatment prior to $\mathrm{P}$. sajor-caju spawn inoculation. In contrast, the amount of lignin was decreased from GM to MSM, which was $22.40 \% \pm 0.34$. This result showed a total decrease of $22.46 \%$ of lignin amount after 5 th harvest of $P$. sajorcaju and thus, indicated that the lignin in GM was partially degraded by P.sajor-caju during the period of cultivation.

The amount of hemicelluloses and cellulose in MSM was lower than GM and RSD. It was considered that this mushroom species also degraded hemicelluloses and cellulose as well as lignin simultaneously. In addition, it had been found that the amount of ash and extractives contents in MSM gave the highest value of $8.10 \% \pm 0.21$ and $12.67 \% \pm 0.18$ respectively, implying that carbohydrate and lignin degradation products of low molecular weight were produced by the extracellular enzymes of $P$. sajor-caju during the cultivation. These results of lignocellulosic composition were consistent with previously reported works in the utilization of different type of mushroom waste medium to yield sugars for ethanol production. As for example, Hiyama et al. obtained an amount of lignin decreased from $24.3 \%$ to $17.8 \%$ after 85 days of shiitake mushroom cultivation on a medium consisted of several woods. The usage of several wood powders as shiitake medium instead of wood $\log$ and period of cultivation might contribute to the difference in amount of lignin removal found in both works [11]. From another previous work performed by Irawati et al, using 77 days-spent culture medium of Auricularia polytricha (black jelly mushroom) cultivated in a medium mixture containing hardwood meal of Alnus japonica, they observed a decrease in lignin, holocellulose and cellulose content from 23.2 to $11.6 \%, 81.1$ to $62.7 \%$, and 44.3 to $31.7 \%$ respectively. In addition, the lignin, hemicellulose, and cellulose content in spent culture medium of softwood (Cryptomeria japonica) gave a decrease from 32.9 to $22.9 \%, \quad 78.2$ to $60.3 \%$, and 51.6 to $36.5 \%$ correspondingly. These results indicated that lignin in the hard- and softwood was partially degraded by A. polytricha during the cultivation period. The amount of lignin and holocellulose in both woodmeal were lower compared to spent culture medium. Thus, indicated that apart of lignin, this mushroom type also degraded cellulose and hemicelluloses during the cultivation [12].

In the utilization of lignocellulosics materials to produce sugars for ethanol fermentation by enzymatic saccharification, lignin would interfered with hydrolysis by blocking the accessibility of cellulase to cellulose [13]. Hence, the removal of lignin from the materials during aforementioned mushroom cultivation could dramatically increase the hydrolysis rate [13], [14], [15]. One of the reasons for high hydrolysis rate was due to the high value of carbohydrate/lignin ratio [16]. According to the previous table of sample composition analysis, the ratio of carbohydrate/ lignin of MSM showed the highest. The cellulose/lignin ratio of RSD, GM, and MSM were 1.15, 1.17, and 1.24 respectively. Collectively, the lignocellulosic components characterization of MSM indicated that such medium have better potential for enzymatic saccharification than RSD and GM due to its low lignin content that would resulted in easy access of the cellulase towards the available amount of cellulose. However, additional pretreatment method such as chemical and thermochemical could be employed on MSM to remove the remaining lignin content to obtain high glucose yield in subsequent enzymatic saccharification.

Table 3.1: Chemical components of RSD, GM, and MSM in percentage (dry weight)

\begin{tabular}{lccccc}
\hline Ash & $\begin{array}{c}\text { Extracti } \\
\text { ves }\end{array}$ & $\begin{array}{c}\text { Hemicellul } \\
\text { ose }\end{array}$ & Lignin & Cellulose \\
\hline RS & $1.87 \pm$ & $3.45 \pm$ & $31.52 \pm$ & $29.30 \pm$ & $33.86 \pm$ \\
D & 0.14 & 0.11 & 0.69 & 1.31 & 1.17 \\
GM & $1.90 \pm$ & $3.78 \pm$ & $31.23 \pm$ & $29.07 \pm$ & $34.02 \pm$ \\
& 0.18 & 0.17 & 1.09 & 0.72 & 0.52 \\
MS & $8.10 \pm$ & $12.67 \pm$ & $27.93 \pm$ & 22.40 & $27.97 \pm$ \\
M & 0.21 & 0.18 & 0.28 & \pm 0.34 & 0.52 \\
\hline
\end{tabular}




\subsection{Surface morphology of RSD, GM and SRSD}

The morphological changes that were induced by biological pretreatment during the period of cultivation of $P$. sajor-caju were examined by Scanning Electron Microscope (SEM) to obtain insight into the structural modification of the raw rubberwood sawdust. The SEM images of MSM were compared with RSD and GM to observe the relationship between the changes in the structural properties at different stages of oyster mushroom cultivation. The morphology surface of untreated RSD, GM, and MSM are shown in Figure 4.1. The biologically untreated RSD and GM showed an even and smooth, flat surface, indicating a rigid and highly ordered surface structure (Figure 3.1 (a) (b)). Regardless the steaming process performed on the GM, its surface structure was not differed much compared to RSD. Additionally, in both sample types, there were traces of small holes that might originated from the previous furnishing and lumbering process of the rubber tree. On the other hand, the MSM had a rugged, rough, and broken surface (Figure 3.1 (c)). The fragments were separated from the initial connected structure and fully exposed, thus increasing the external surface area and porosity resulting in loose structure of the fibre bundles. This would obviously favour the enzymes contacting the inner linkage, hence accelerating the saccharification process. The SEM figures of the MSM also clearly showed traces of hyphae on the fibe surface resulting from the oyster mushroom cultivation. Taniguchi et al. also discovered a loosening structural of cells with a simultaneous increase in porosity of rice straw resulted from cultivation of $P$. ostreatus for a period of 36 and 60 days. The phenomenon was due to the partial degradation of the lignin that was responsible for preventing the penetration of cellulase in the rice straw in enzymatic saccharification [17].

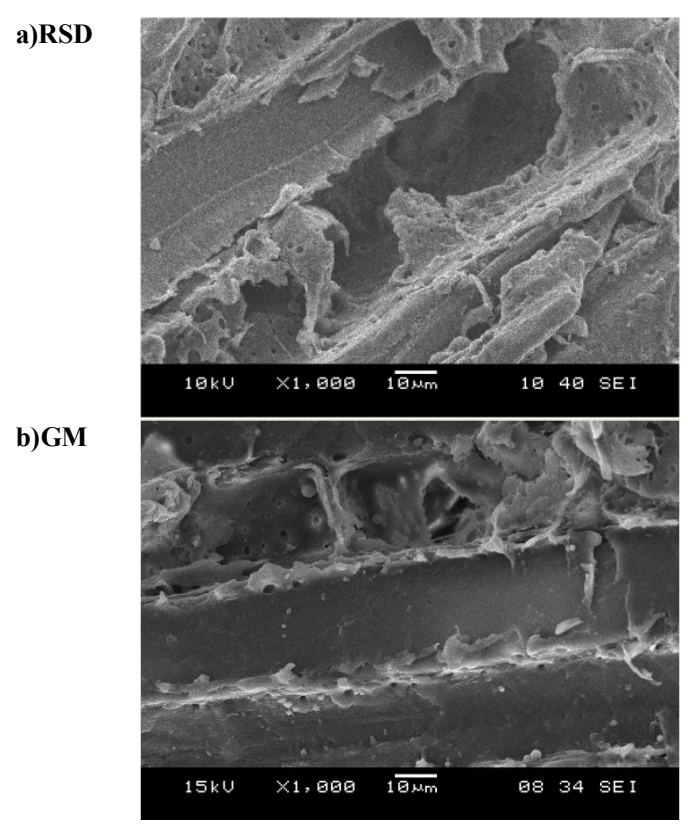

c)RSD

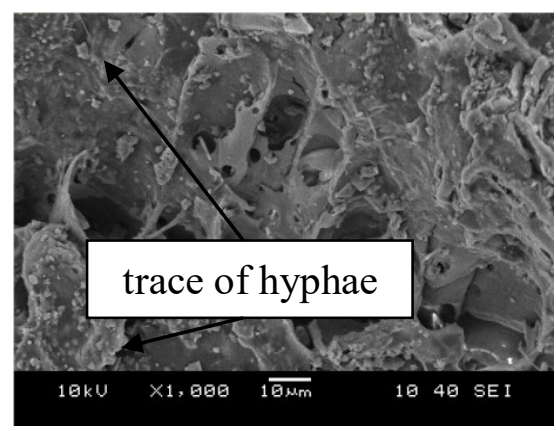

Figure 3.1: Images of morphology structure of (a) RSD, (b) GM, and (c) MSM at magnification of $1000 \mathrm{x}$

\subsection{Glucose yield}

Figure 3.2 shows the amount of glucose obtained from RSD, GM, and MSM after $\mathrm{NaOH}$ pretreatment via autoclaving method at $121^{\circ} \mathrm{C}$ for $20 \mathrm{~min}$. Based on the results of the work, it showed that, the $\mathrm{NaOH}$ treatment improved most of the fiber properties as the glucose yield obtained from the three different samples increased with pretreatment severity. The highest glucose yield was resulted from $\mathrm{NaOH}$ pretreatment at $2.0 \mathrm{M}$ for all three samples. The yields of glucose of RSD, GM, and MSM under the employed pretreatment condition were 21.97, 19.81, and $31.07 \mathrm{~g} / 100 \mathrm{~g}$ dry substrate respectively. The applied pressure at elevated temperature and high concentration of $2.0 \mathrm{M}$ of $\mathrm{NaOH}$ during autoclaving process had loosened the structure of RSD, GM, and MSM, and increased the degree of delignification. The effect could be clearly seen from the increment of glucose yield towards the increasing of the applied $\mathrm{NaOH}$ concentration. The yield of glucose based on $\mathrm{NaOH}$-treated MSM was markedly better compared to the original untreated MSM. Compared to untreated $\mathrm{MSM}$, the $2.0 \mathrm{M} \mathrm{NaOH}$ treated-MSM gave an increase of 3.32 fold of glucose yield. This result indicated that, the combination of biological and thermo-chemical pretreatment on MSM could improve the glucose yield. In a study performed by McIntosh and Vancov in $\mathrm{NaOH}$ $(0.5 \mathrm{M})$ pretreatment of sorghum straw (Sorghum bicolour var. MR Buster) via autoclaving at $121^{\circ} \mathrm{C}$ with temperature of $60^{\circ} \mathrm{C}$, a 5.6 fold increase in total of reducing sugars was obtained in comparison to samples treated at the same temperature in the absence of $\mathrm{NaOH}$. The result confirmed the importance of alkali inclusion to adequately remove a certain amount of lignin for high production of reducing sugars [18]. Meanwhile, Hamzah et al. has treated the oil palm (Elaeis) empty fruit bunches fiber (EFB) with $2.5 \mathrm{~mol} / \mathrm{L} \mathrm{NaOH}$ for $15 \mathrm{~min}$. They obtained a significant reduction in hemicelluloses and lignin amount from 35 to 24.55 and 16.45 to 7.25 $\mathrm{g} / 100 \mathrm{~g}$ EFB fiber in comparison to the untreated EFB, resulting in high amount of cellulose portion of 66.77 $\mathrm{g} / 100 \mathrm{~g}$ EFB fiber. They suggested that, the combination of the $\mathrm{NaOH}$ swelling effect and high temperature disintegrated the EFB fibre into a pliable fiber, cleaned up the fiber surface, and thus exposed more cellulose component in the EFB fibre [19]. Additionally, 
according to Astimar et al., a part of effectively removing hemicelluloses and lignin, the $\mathrm{NaOH}$ also penetrated and swelled both the accessible amorphous and crystalline regions of cellulose [20].

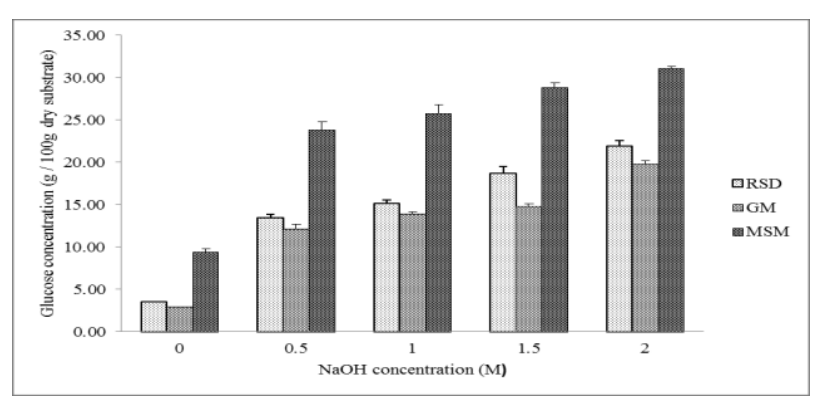

Figure 3.2: Amount of glucose (g/100 g dry substrate) of different concentration NaOH-treated RSD, GM, and MSM (autoclaving method)

Figure 3.3 shows that increasing temperature and $\mathrm{NaOH}$ concentration could improve the production of glucose after enzymatic saccharification of RSD, GM, and MSM. As shown in the corresponding figures, treating MSM in $2.0 \mathrm{M} \mathrm{NaOH}$ at $30^{\circ} \mathrm{C}, 60^{\circ} \mathrm{C}$, and $90^{\circ} \mathrm{C}$ had enhanced the glucose yield by $8.45,3.63$, and 3.77 fold compared to untreated MSM. In comparison to glucose yield obtained after autoclaving method using $2.0 \mathrm{M} \mathrm{NaOH}$, the glucose yield resulted from heating in a water bath at $90^{\circ} \mathrm{C}$ with similar $\mathrm{NaOH}$ concentration was $30.13 \mathrm{~g} / 100 \mathrm{~g}$ dry substrate, which was slightly higher. Treating the MSM sample in water bath at 30 and $60^{\circ} \mathrm{C}$ gave an amount of glucose yield of 23.63 and $26.53 \mathrm{~g} / 100 \mathrm{~g}$ dry substrate after $48 \mathrm{~h}$ of enzymatic saccharification. Under all pretreatment conditions of different temperature and $\mathrm{NaOH}$ concentration, the glucose yield obtained from RSD and GM was comparable. This indicated that the steaming process on GM before spawn inoculation did not improve and affected much the fiber properties for the feasibility of cellulase susception in enzymatic saccharification. The direct enzymatic saccharification of RSD, GM, and MSM without inclusion of $\mathrm{NaOH}$ treatment was ineffective because lignin surrounding cellulose prevented the enzymes from accessing cellulose. However, the glucose yields resulted from MSM at treatment temperature of 30,60 , and $90^{\circ} \mathrm{C}$ were the highest between the three samples type, which were $2.79,7.30$, and $7.99 \mathrm{~g} / 100 \mathrm{~g}$ dry substrate, approximately $3.58,3.08,2.14$ fold of increment, respectively compared to RSD. This might be due to the feasibility of cellulase to access the cellulose as the amount of lignin removed increased with pretreatment temperature and $\mathrm{NaOH}$ concentration. In addition, the amount of glucose resulted from untreated MSM was comparable to those obtained from $1.0 \mathrm{M}$ of $\mathrm{NaOH}$ treated-RSD at $60^{\circ} \mathrm{C}$.
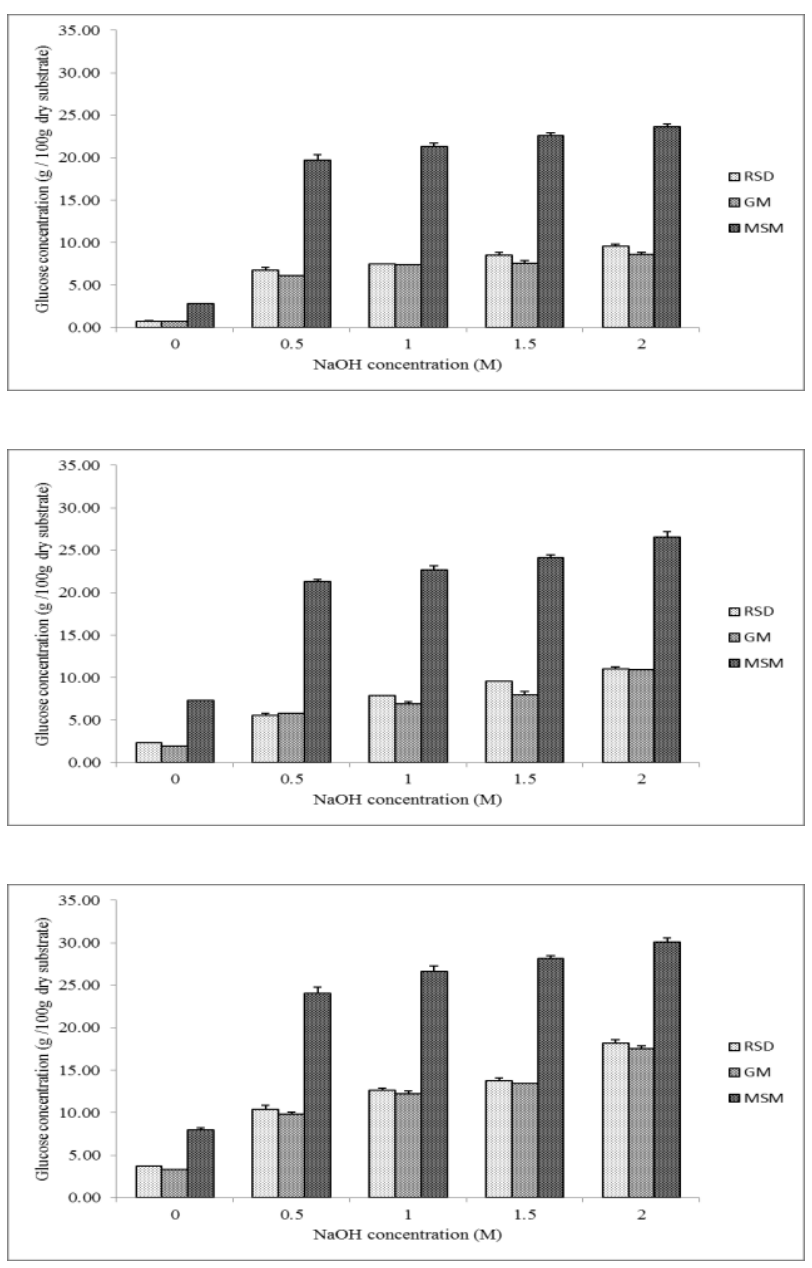

Figure 3.3: Amount of glucose ( $\mathrm{g} / 100 \mathrm{~g}$ dry substrate) of different concentration $\mathrm{NaOH}$-treated RSD, GM, and MSM (heating method at a) 30, b) 60, c) $90^{\circ} \mathrm{C}$ )

The amount of glucose obtained from different pretreated RSD, GM, and MSM soaked in various concentrations of $\mathrm{NaOH}$ pretreament at room temperature are depicted in Figure 3.4. From the figure, the pretreatment condition of soaking MSM in $2.0 \mathrm{M}$ $\mathrm{NaOH}$ gave the highest yield of glucose with approximately 2.93 fold compared to RSD. In comparison to the treated MSM in distilled water, the 2.0 $\mathrm{M} \mathrm{NaOH}$-treated MSM showed an increase of 10.12 fold of glucose yield. This indicated that the amount of lignin removal after oyster mushroom cultivation increased with the increase in $\mathrm{NaOH}$ concentration employed at ambient temperature. The enzymatic saccharification of treated RSD and GM without $\mathrm{NaOH}$ inclusion was ineffective because lignin and hemicelluloses surrounding cellulose prevented the enzymes from accessing the cellulose. As for MSM, soaking in distilled water at room temperature was not enough to remove the remaining hyphen and lignin that formed a barrier between cellulase and cellulose. However, after $\mathrm{NaOH}$ inclusion, the glucose yield obtained increased with increase in $\mathrm{NaOH}$ concentration used for all types of samples, implying that the amount of lignin and hemicelluloses removed also increased. The amount of 
glucose obtained from GM under all concentration of $\mathrm{NaOH}$ pretreatment showed only a slightly different from RSD regardless the previous performed steaming process. It could be concluded that, the combination of biological and thermo-chemical pretreatment on MSM could markedly improve the glucose yield in enzymatic saccharification rather to employ only one separate pretreatment. Therefore, the pretreatment at room temperature only resulted in low yields of glucose.

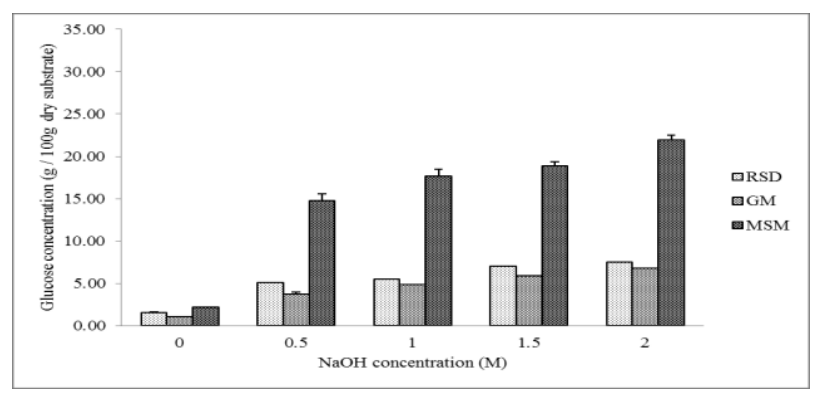

Figure 3.4: Amount of glucose (g/100g dry substrate) of different concentration $\mathrm{NaOH}$-treated RSD, GM, and MSM (soaking method)

\section{Conclusion}

From the results of lignocellulosic composition and morphology surface analysis of RSD, GM, and MSM, it can be concluded that the yield of glucose produced in enzymatic saccharification can be attributed to three factors: (1) the crystallinity of cellulose, (2) the ligninhemicellulose sheath that surrounds the cellulose, and (3) the availability of surface area for the enzyme attack. The study showed that MSM was a promising biomass material for enzymatic saccharification to produce bioethanol or other bioproducts as well considering its high glucose yield in comparison to RSD and GM. Under all employed pretreatment techniques, the highest yield of glucose and hydrolysis weight decreases were obtained from MSM. The high glucose production obtained via heating in a water bath at $90^{\circ} \mathrm{C}$ for $2 \mathrm{~h}$ with 2.0 $\mathrm{M} \mathrm{NaOH}$ proved that the combination of biological and thermo-chemcial pretreatment was effective to reduce the lignin and increase the cellulose content in the MSM. However, increase in thermo-chemical pretreatment severity until certain limit would increase the amount of lignin removed as well as the glucose produced. Under more severerer pretreatment conditions, which lead to greater lignin reductions, did not favour the glucose production due to substantial amount of solid losses.

\section{References}

1. Pan, X., Arato, C., Gilkes, N., D.Gregg, \& Mabee, W. Biotechnol. Eng., 90, 473-481, (2005).

2. Srinivasakannan, C., \& Bakar, M. Z. A. Biomass Bioenergy, 27, 89-96. (2004).

3. Alhassan, A. M. University of Putra Malaysia, Serdang, Selangor. (2010).

4. Wan, C., \& Li, Y. Enzym and Microbial Technology, 47, 31-36. (2010).

5. Koshy, J., \& Nambisan, P. International Journal of Applied Biology and Pharmaceutical Technology, 3(1), 280-286. (2012).

6. Punsuvon, V. [Electronic Version], 157-178. (2013).

7. Ramsden, M. J., \& Blake, F. S. R. Wood Science and Technology, 31, 45-50. (1997).

8. Li, S., Xu, S., Liu, S., Yang, C., \& Lu, Q. Fuel Processing Technology, 85, 1208-1211. (2004).

9. William E. Kaar, M. T. H. Biomass and Bioenergy, 18, 198-199. (2000).

10. Neureiter G. L., H. Danner, C. Thomasser, B. Saidi, \& Braun., R. Appli. Biochem. Biotechnol, 98, 49-58. . (2002)

11. Hiyama, R., Gisusi, S., \& Harada, A. J Wood Sci, 57, 429-435. (2011)

12. Irawati, D., Yokota, S., Niwa, T., Takashima, Y., Ueda, C., Ishiguri, F. J Wood Sci, 58, 180183.(2012)

13. Cheng, C.-L., Lo, Y.-C., Lee, K.-S., Lee, D.-J., Lin, C.-Y., \& Chang, J.-S. Bioresource Technology, 102(18), 8514-8523. (2011).

14. Lee, J.-W., Koo, B.-W., Choi, J.-W., Choi, D.H., \& Choi, I.-G. Bioresource Techn, 99, 27362741. (2008)

15. Yokota, S., Nakajima, R., Suzuki, D., Ishiguri, F., Iizuka, K., \& Yoshizawa, N. Cellulose Chem Technol, 41, 575-582. (2007).

16. Xu, J., Chen, Y., Cheng, J. J., SharmaShivappa, R. R., \& Burns, J. C. Bioresources, 6(1). (2011).

17. Taniguchi, M., Suzuki, H., Watanabe, D., Sakai, K., Hoshino, K., \& Tanaka, T. Journal of Bioscience and Bioengineering, 100(6), 637643. (2005).

18. McIntosh, S., \& Vancov, T. Bioresource Technology, 101(17), 6718-6727. (2010).

19. Hamzah, F., Idris, A., \& Shuan, T. K. Biomass and Bioenergy, 35(3), 1055-1059. (2011).

20. Astimar, A.A., Das. K., Husin. M. \& Anis. M. J. Oil Palm Research. Vol. 14, No. 2, 10-17. (2002). 